\title{
Quantum efficiency and thermal emittance of metal photocathodes
}

\author{
David H. Dowell and John F. Schmerge \\ SLAC, Menlo Park, California 94025, USA \\ (Received 2 May 2009; published 27 July 2009)
}

\begin{abstract}
Modern electron beams have demonstrated the brilliance needed to drive free electron lasers at x-ray wavelengths with major advances occurring since the invention of the photocathode gun and the realization of emittance compensation. These state-of-the-art electron beams are now becoming limited by the intrinsic thermal emittance of the cathode. In both $\mathrm{dc}$ and $\mathrm{rf}$ photocathode guns details of the cathode emission physics strongly influence the quantum efficiency and the thermal emittance. Therefore improving cathode performance is essential to increasing the brightness of beams. It is especially important to understand the fundamentals of cathode quantum efficiency and thermal emittance. This paper investigates the relationship between the quantum efficiency and the thermal emittance for metal cathodes using the Fermi-Dirac model for the electron distribution. We use a consistent theory to derive the quantum efficiency and thermal emittance, and compare our results to those of others.
\end{abstract}

DOI: 10.1103/PhysRevSTAB.12.074201

PACS numbers: 07.77.Ka, 52.59.-f, 71.10.Ca, 85.60.Ha

\section{INTRODUCTION}

In this paper we use the three-step photoemission model of Puff [1] and Spicer [2,3] to obtain expressions for the quantum efficiency and the uncorrelated or thermal emittance of metal cathodes. In this model, the first step is the absorption of the incident photon by an electron described by the two important phenomena of reflectivity and absorption as the photons travel into the cathode. The second step contains the physics occurring while the electron drifts to the surface. Here the relevant effects are electronelectron scattering and the angular cone of escaping electrons. In the third step the important phenomena are the Schottky effect and the abrupt change in electron angle across the metal-vacuum interface.

Previously we developed analytic expressions for the quantum efficiency and thermal emittance based on the three-step model ignoring electron-electron scattering [4]. Further work [5] expanded the quantum efficiency model to include electron-electron scattering, resulting in excellent agreement with the measured quantum efficiency of an atomically clean copper surface. Here we apply the same Fermi-Dirac distribution with the three-step model of photoemission to give a consistent theory of the emittance and the quantum efficiency. Other recent work [6] generalizes this basic phenomenological process to give a common emission theory for thermionic, photoelectric, and field emission. Here we concentrate on the connection between quantum efficiency and the photoelectric thermal emittance.

The derivation for the quantum efficiency (QE) and the thermal emittance begins with the electron gas theory for metals and Spicer's three-step model for photoemission as illustrated in Fig. 1. In a previous publication [5], we calculated the QE using this approach using the work function as the only free parameter. In this model, the electron is emitted by means of three sequentially independent processes: (1) absorption of the photon with energy $\hbar \omega$, (2) migration, including $e-e$ scattering, to the surface, and (3) escape for electrons with kinematics above the barrier. Here the same methodology is used to derive the thermal emittance for metal photocathodes.

In the next section the quantum efficiency is obtained from Fermi-Dirac statistics for fermions. This is followed by a derivation of the thermal or photoelectric emittance. In the last two sections, the relationship between the cathode emittance and the quantum efficiency is discussed for metallic emitters, and the results are compared with previously published work.

\section{QUANTUM EFFICIENCY AND THE FERMI- DIRAC MODEL FOR METALS}

Being fermions, electrons uniformly fill all energy states up to the Fermi level, $E_{F}$, with no more than two opposing spin electrons per energy state. Therefore electron-electron scattering below the Fermi level is strongly suppressed due

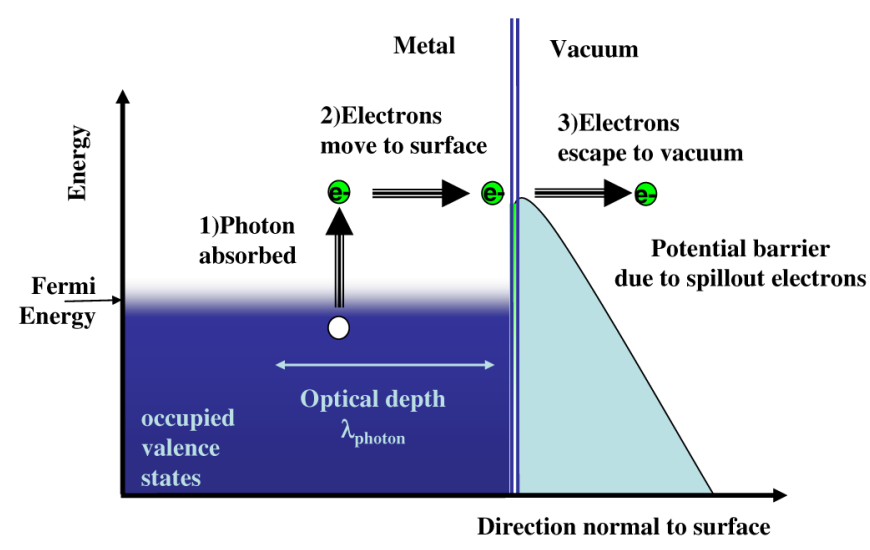

FIG. 1. (Color) Three-step model of photoemission. 
to the Pauli exclusion principle and the lack of unoccupied final states, also known as Pauli blocking. As a result, electrons move freely through the metal with long meanfree paths which contributes to the good electrical conductivity of metals. The distribution of occupied states as a function of energy is given by the Fermi-Dirac function,

$$
f_{\mathrm{FD}}(E)=\frac{1}{1+e^{\left(E-E_{F}\right) / k_{B} T}},
$$

where $k_{B} T$ is the electron gas thermal energy, $E_{F}$ is the Fermi energy, and $E$ is the state energy. To be more precise, the Fermi energy in Eq. (1) should be replaced by the chemical potential or Fermi level. However, at low temperatures the Fermi energy and chemical potential are nearly identical and at absolute zero they are identical. In this paper we only consider low temperatures and thus the use of the Fermi energy is appropriate.

The electron density of occupied states inside the cathode and the electric potentials experienced by a single electron immediately outside the cathode are plotted in Fig. 2. The occupation number is shown for a Fermi-
Dirac function at finite temperature. The Schottky potential is the sum of the image charge field and the applied field, $F_{a}$. The Schottky work function, $\phi_{\text {Schottky }}$, is the peak value of the Schottky potential and is the height of the photoemission potential barrier typically located a few nanometers outside the cathode. The zero field vacuum state is shifted downward at high electric field by the Schottky work function reducing the barrier and increasing the quantum yield. The effective work function, $\phi_{\text {eff }}$, is defined as

$$
\begin{aligned}
\phi_{\text {eff }} & \equiv \phi_{w}-\phi_{\text {Schottky }}=\phi_{w}-e \sqrt{\frac{e F_{a}}{4 \pi \varepsilon_{0}}} \\
& =\phi_{w}-0.037947 \sqrt{F_{a}(\mathrm{MV} / \mathrm{m})} \mathrm{eV} .
\end{aligned}
$$

Incident photons with energies above the effective work function are absorbed by electrons near the Fermi energy which then migrate to the surface and escape.

The QE can be expressed in terms of the probabilities for these steps to occur where it is assumed $\hbar \omega \leq E_{F}$,

$$
\mathrm{QE}(\omega)=[1-R(\omega)] \frac{\int_{E_{F}+\phi_{\mathrm{eff}}-\hbar \omega}^{\infty} d E\left[1-f_{\mathrm{FD}}(E+\hbar \omega)\right] f_{\mathrm{FD}}(E) \int_{\cos \theta_{\max }(E)}^{1} d(\cos \theta) F_{e-e}(E, \omega, \theta) \int_{0}^{2 \pi} d \Phi}{\int_{E_{F}-\hbar \omega}^{\infty} d E\left[1-f_{\mathrm{FD}}(E+\hbar \omega)\right] f_{\mathrm{FD}}(E) \int_{-1}^{1} d(\cos \theta) \int_{0}^{2 \pi} d \Phi}
$$

Here $R(\omega)$ is the cathode optical reflectivity. The FermiDirac function, $f_{\mathrm{FD}}(E)$, is the density of initial states, and $\left[1-f_{\mathrm{FD}}(E+\hbar \omega)\right]$ is the density of final states with the product of these two functions giving the transition probability for the excited electron to escape. $F_{e^{-e} e}(E, \omega, \theta)$ is the probability the excited electron reaches the surface without scattering. This function is determined by the photon ab- sorption length, $\lambda_{\mathrm{opt}}$, and the electron-electron mean-free path, $\lambda_{e-e}$. The coordinate system is shown in Fig. 5.

Unlike a semiconductor photocathode, in a metal electron-phonon scattering can be ignored with electronelectron scattering dominating. Since the photon energy is less than twice the work function, any electron-electron scattering event eliminates both electrons from the possi-

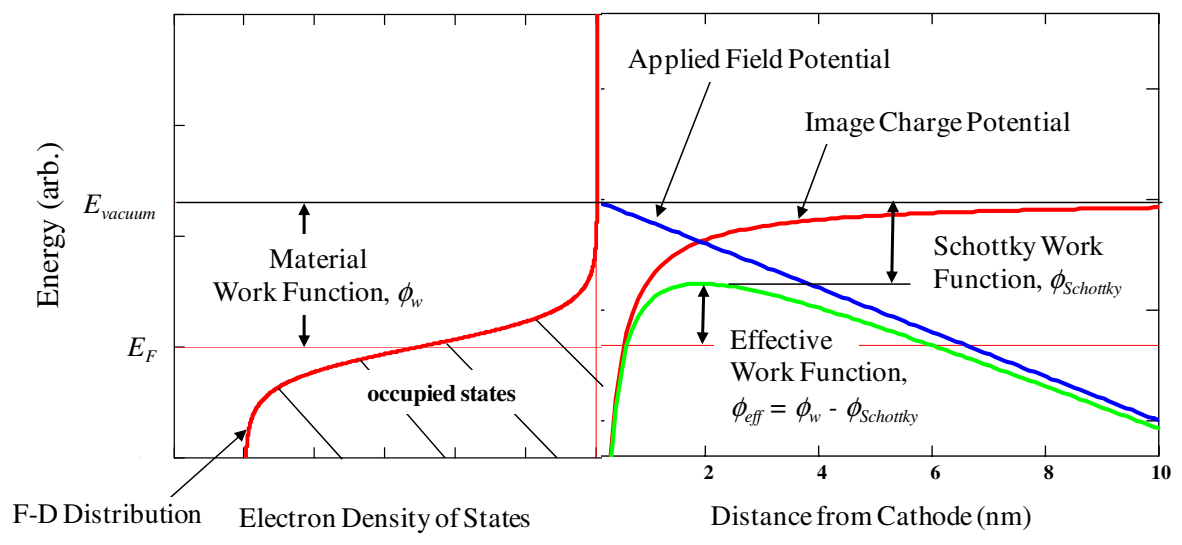

FIG. 2. (Color) The energy distribution of occupied states for a metal (left) and the electric potentials next to the cathode surface (right). The Schottky potential (green curve) is the sum of the applied field potential (blue) and the image charge potential (red). The Schottky work function is the amount the material work function is reduced at the peak of the Schottky potential. The Schottky potential is shown for an applied field of $100 \mathrm{MV} / \mathrm{m}$ where the peak of the photoemission barrier is $1.9 \mathrm{~nm}$ from the surface. The effective work function is the height of this barrier above the Fermi level and is the material work function reduced by the Schottky work function. 
bility of escaping. Hence, the fraction of electrons per unit distance at a depth $s$ below the surface which survive scattering is

$$
f(s, E, \theta, \omega)=\frac{1}{\lambda_{\mathrm{opt}}(\omega)} e^{-s\left(\left[1 / \lambda_{\mathrm{opt}}(\omega)\right]+\left\{1 /\left[\lambda_{e^{-} e}(E) \cos \theta(E)\right]\right\}\right)} .
$$

Here we explicitly show the complete functional dependence of the scattering probability upon the photon wavelength, the electron energy, and the angle of the electron's motion relative to the surface normal. For the situation of photoemission close to the barrier, i.e., where $\hbar \omega$ - $\phi_{\text {eff }}$ is a couple of tenths of an $\mathrm{eV}$, the electron-electron mean-free path is nearly constant over this small energy range and its average value can be used. This is evident in Fig. 7 of Ref. [5]. In addition, the maximum angle the electron can have when it reaches the surface and can escape, $\theta_{\max }(E)$ which is defined below, is typically 20 degrees and the angular dependence can be ignored. Thus, to a good approximation only the wavelength dependence remains, and the $s$ integration of Eq. (4) gives the following for the probability $F_{e-e}$ :

$$
\begin{aligned}
F_{e^{-e}}(\omega) & =\int_{0}^{\infty} f(s, \theta=0, \omega) d s \\
& =\int_{0}^{\infty} \frac{1}{\lambda_{\mathrm{opt}}(\omega)} e^{\left.\left.-s\left[1 / \lambda_{\mathrm{opt}}(\omega)\right]+\left(1 / \bar{\lambda}_{e^{-} e}\right)\right]\right\}} d s \\
& =\frac{1}{1+\frac{\lambda_{\mathrm{opt}}(\omega)}{\bar{\lambda}_{e^{-} e}}} .
\end{aligned}
$$

The energy dependence of the average mean-free path can be expressed in terms of a measured mean-free path, $\lambda_{m}$, at the energy, $E_{m}$, relative to the Fermi energy [5],

$$
\bar{\lambda}_{e-e}(\omega)=\frac{\int_{\phi_{\mathrm{eff}}}^{\hbar \omega} \lambda_{e-e}(E) d E}{\int_{\phi_{\mathrm{eff}}}^{\hbar \omega} d E}=\frac{2 \lambda_{m} E_{m}^{3 / 2}}{\hbar \omega \sqrt{\phi_{\mathrm{eff}}}} \frac{1}{\left(1+\sqrt{\frac{\phi_{\mathrm{eff}}}{\hbar \omega}}\right)}
$$

The effect of neglecting both the angular dependence of the scattering probability and the energy dependence of the mean-free path is justified later in this section. With these assumptions, the electron-electron scattering probability depends only upon $\omega$ allowing it to be moved outside the integrals and the equation for the $\mathrm{QE}$ becomes

$$
\mathrm{QE}(\omega)=[1-R(\omega)] F_{e^{-} e}(\omega) \frac{\int_{E_{F}+\phi_{\mathrm{eff}}-\hbar \omega}^{\infty} d E\left[1-f_{\mathrm{FD}}(E+\hbar \omega)\right] f_{\mathrm{FD}}(E) \int_{\cos \theta_{\max }(E)}^{1} d(\cos \theta) \int_{0}^{2 \pi} d \Phi}{\int_{E_{F}-\hbar \omega}^{\infty} d E\left[1-f_{\mathrm{FD}}(E+\hbar \omega)\right] f_{\mathrm{FD}}(E) \int_{-1}^{1} d(\cos \theta) \int_{0}^{2 \pi} d \Phi}
$$

The photon absorption in the three-step model conserves energy but not momentum or otherwise only electrons moving away from the surface would be excited by photons at normal incidence and the photoemission yield would be vanishingly small. This assumption of momentum nonconservation has a long history and was discussed extensively by Spicer during the formative years of the three-step model $[1,2,7,8]$, where the optical absorption of the photon is considered to be direct if the momentum is conserved and indirect if not. Indirect optical transitions would involve momentum conservation via multistep absorption. For example, after absorbing the photon the electron elastically scatters from phonons which randomize its momentum [9].

Assuming the electrons in a metal behave as noninteracting fermions, they occupy states as given by the FermiDirac distribution, $f_{\mathrm{FD}}(E)$. In the above equation, $f_{\mathrm{FD}}(E)$ is the initial state occupation number as a function of electron energy. $f_{\mathrm{FD}}(E+\hbar \omega)$ is the final state occupation number vs energy for electrons which absorb a photon. At low thermal energy or when $k_{B} T \ll E_{F}$, the Fermi-Dirac distribution is well represented by the Heaviside-step function, $H$. Numerical results with nonzero temperatures are described later. Although the actual density of states is not constant over all energies, in metals at energies close to the Fermi energy the step function approximation is still valid because electrons are only emitted from a small energy range where the density of states can be approximated with a flat distribution. For applications where the photon energy significantly exceeds the effective work function, other energy states can be excited and this approximation may not be valid. With these assumptions, the energy integration limits for transition probability between initial and final states becomes

$$
\int_{E_{F}+\phi_{\mathrm{eff}}-\hbar \omega}^{\infty}\left[1-f_{\mathrm{FD}}(E+\hbar \omega)\right] f_{\mathrm{FD}}(E) d E=\int_{E_{F}+\phi_{\mathrm{eff}}-\hbar \omega}^{\infty}\left[1-H\left(E_{F}-E-\hbar \omega\right)\right] H\left(E_{F}-E\right) d E=\int_{E_{F}+\phi_{\mathrm{eff}}-\hbar \omega}^{E_{F}} d E
$$

with the equation for the $\mathrm{QE}$ reducing to

$$
\mathrm{QE}(\omega)=[1-R(\omega)] F_{e-e}(\omega) \frac{\int_{E_{F}+\phi_{\mathrm{eff}}-\hbar \omega}^{E_{F}} d E \int_{\sqrt{\left(E_{f}+\phi_{\mathrm{eff}} /(E+\hbar \omega)\right.}}^{1} d(\cos \theta) \int_{0}^{2 \pi} d \Phi}{\int_{E_{F}-\hbar \omega}^{E_{F}} d E \int_{-1}^{1} d(\cos \theta) \int_{0}^{2 \pi} d \Phi}
$$


Equation (9) can be integrated to obtain

$$
\mathrm{QE}(\omega)=\frac{1-R(\omega)}{1+\frac{\lambda_{\mathrm{opt}}(\omega)}{\bar{\lambda}_{e^{-e}}(\omega)}} \frac{\left(E_{F}+\hbar \omega\right)}{2 \hbar \omega}\left[1-\sqrt{\frac{E_{F}+\phi_{\mathrm{eff}}}{E_{F}+\hbar \omega}}\right]^{2}
$$

The QE can be expanded as a function of $\hbar \omega-\varphi_{\text {eff }}$ in the following Taylor series which is evaluated at photoemission threshold where $\hbar \omega$ is only slightly larger than $\phi_{\text {eff }}$ :

$$
\begin{aligned}
\mathrm{QE}(\omega)= & \left.\mathrm{QE}\right|_{\hbar \omega=\phi_{\mathrm{eff}}}-\left.\frac{d \mathrm{QE}}{d \hbar \omega}\right|_{\hbar \omega=\phi_{\mathrm{eff}}}\left(\hbar \omega-\phi_{\mathrm{eff}}\right) \\
& +\left.\frac{1}{2} \frac{d \mathrm{QE}}{d \hbar \omega}\right|_{\hbar \omega=\phi_{\mathrm{eff}}}\left(\hbar \omega-\phi_{\mathrm{eff}}\right)^{2}-\cdots
\end{aligned}
$$

The first two terms are zero verifying the well-known quadratic dependence upon the photon energy for the quantum efficiency near threshold result [10]:

$$
\mathrm{QE}(\omega) \approx \frac{1-R(\omega)}{1+\frac{\lambda_{\mathrm{opt}}(\omega)}{\lambda_{e^{-} e}(\omega)}} \frac{\left(\hbar \omega-\phi_{\mathrm{eff}}\right)^{2}}{8 \phi_{\mathrm{eff}}\left(E_{F}+\phi_{\mathrm{eff}}\right)} .
$$

As described earlier, multiple assumptions are required to produce this analytic result. Therefore we also numerically integrate the above equations to test the accuracy of several of the assumptions. Two assumptions were necessary to arrive at the average mean-free path shown in Eq. (6). The first assumption was to ignore the $E^{-3 / 2}$ energy dependence of the mean-free path [5]. For the parameters listed in Table I, the mean-free path energy dependence results in a 3.2\% reduction in QE. The second assumption was to approximate the electron path length with the shortest distance from the point of absorption to the surface. In other words, the angular dependence of the scattering probability was ignored. Once again for the parameters listed in Table I, the angular dependence of the scattering probability results in less than $1 \%$ reduction in QE. The reason these two assumptions have little effect on the calculated QE is because the difference between the photon energy and effective work function is small resulting in a narrow energy and angular acceptance band for the emitted electrons. As this difference increases and the permitted energies and angles increase, the approximation will become less accurate.

TABLE I. Physical constants used to compute the thermal emittance and quantum efficiency for atomically clean copper [5].

\begin{tabular}{lc}
\hline \hline Fermi energy, $E_{F}$ & $7 \mathrm{eV}$ \\
Work function, $\phi_{w}$ & $4.31 \mathrm{eV}$ \\
$\phi_{\text {Schottky } @ 50 \mathrm{MV} / \mathrm{m}}$ & $0.268 \mathrm{eV}$ \\
$e-e$ scattering length @ 8.6 eV, $\lambda_{e^{-e}}\left(E_{m}\right)$ & 22 angstroms \\
$E_{m}$ & $8.6 \mathrm{eV}$ \\
Photonenergy @255 nm & $4.86 \mathrm{eV}$ \\
\hline \hline
\end{tabular}

In addition we have assumed the temperature is $0 \mathrm{~K}$ to allow replacing the Fermi-Dirac distribution with the Heaviside-step function. The electron temperature is defined as the temperature of the electrons prior to the absorption of a photon. Numerical integration shows that for the parameters in Table I the electron temperature must be approximately $2000 \mathrm{~K}$ to increase the QE by $10 \%$ and over $7000 \mathrm{~K}$ to increase the QE by a factor of 2 . At ambient temperatures near $300 \mathrm{~K}$ where most photocathodes are operated, the effect on the QE is an approximately $0.1 \%$ increase. The temperature has little effect until the ratio of $k_{B} T /\left(h \omega-\phi_{\text {eff }}\right)$ approaches unity.

We have also assumed a monoenergetic photon beam in the derivation of the $\mathrm{QE}$. The photons can be considered monoenergetic unless the photon energy spread becomes comparable to the electron temperature. Assuming a $255 \mathrm{~nm}$ photon and transform limited pulse, the pulse length must be $<13$ fs to produce greater than $25 \mathrm{meV}$ energy spread. Thus, the energy spread of the photon can be ignored even for pulses with $100 \mathrm{fs}$ rise times.

\section{THE PHOTOELECTRIC EMITTANCE FOR METAL CATHODES}

This section derives the thermal emittance for photoelectric emission by applying the same Fermi-Dirac model as used to obtain the quantum efficiency and closely follows that performed for $\mathrm{Cs}_{2} \mathrm{Te}$ cathodes by Floettmann [11]. However, before launching into the derivation of the emittance it is necessary to discuss the boundary conditions of the escaping electron.

\section{A. The boundary condition and the maximum angle of escape}

Similar to photons, electrons change their angle or refract as they move from inside to outside the cathode. This is a result of the boundary condition requiring conservation of transverse momentum across the cathode-vacuum interface. In terms of the momenta and angles defined in Fig. 3, this boundary condition is expressed as

$$
p_{x}^{\text {in }}=p_{x}^{\text {out }}
$$

or equivalently,

$$
p_{\text {total }}^{\text {in }} \sin \theta_{\text {in }}=p_{\text {total }}^{\text {out }} \sin \theta_{\text {out }} .
$$

The total energy of the electron (after absorbing a photon) inside the cathode is $E+\hbar \omega$. The electron energy outside the cathode is this energy minus the energy of the vacuum state, $E_{F}+\phi_{\text {eff }}$ as shown in Fig. 2, giving its energy after emission as $\left(E+\hbar \omega-E_{F}-\phi_{\text {eff }}\right)$. Thus, the total momentum inside and outside the cathode are

$$
\begin{aligned}
& p_{\text {total }}^{\text {in }}=\sqrt{2 m(E+\hbar \omega)} \text { and } \\
& p_{\text {total }}^{\text {out }}=\sqrt{2 m\left(E+\hbar \omega-E_{F}-\phi_{\text {eff }}\right)}
\end{aligned}
$$




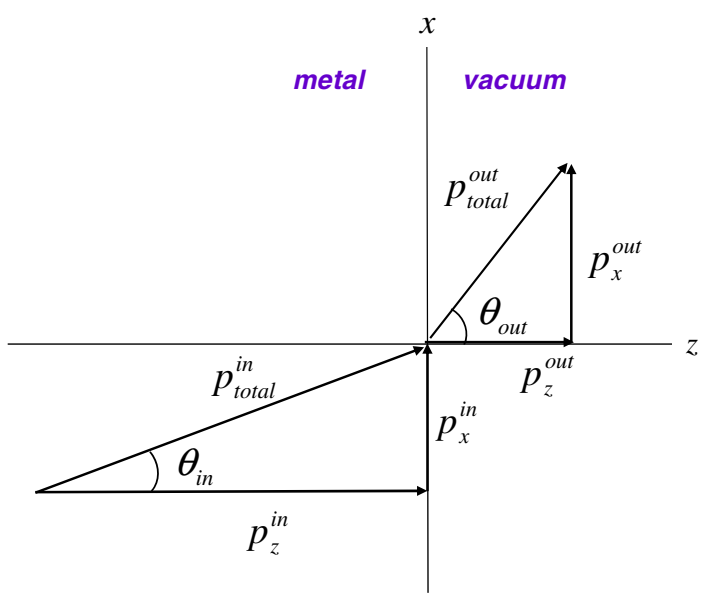

FIG. 3. (Color) Diagram showing the conservation of transverse momentum at the metal-vacuum interface. Outside the surface the maximum angle is $\pi / 2$ when the electrons are refracted with the largest internal angle of incidence $\theta_{\max }^{\text {in }}$. Electrons with angles greater than $\theta_{\max }^{\text {in }}$ are reflected back into the metal.

Inserting these expressions into the above equation for the boundary condition gives a formula similar to Snell's law for optics,

$$
\frac{\sin \theta_{\text {out }}}{\sin \theta_{\text {in }}}=\sqrt{\frac{E+\hbar \omega}{E+\hbar \omega-E_{F}-\phi_{\text {eff }}}} .
$$

An electron inside the cathode approaching the boundary needs to have sufficient momentum normal to the barrier to escape,

$$
p_{z}=\sqrt{2 m(E+\hbar \omega)} \cos \theta_{\text {in }} \geq \sqrt{2 m\left(E_{F}+\phi_{\text {eff }}\right)} .
$$

This leads to the maximum internal angle for which the electron with energy $E$ can escape, $\theta_{\max }^{\text {in }}$,

$$
\cos \theta_{\max }^{\text {in }}=\sqrt{\frac{E_{F}+\phi_{\text {eff }}}{E+\hbar \omega} .}
$$

This relationship between the momenta inside the cathode defining the maximum escape angle is shown graphically in Fig. 4.

The angle on the vacuum side of the boundary corresponding to the internal maximum escape angle is

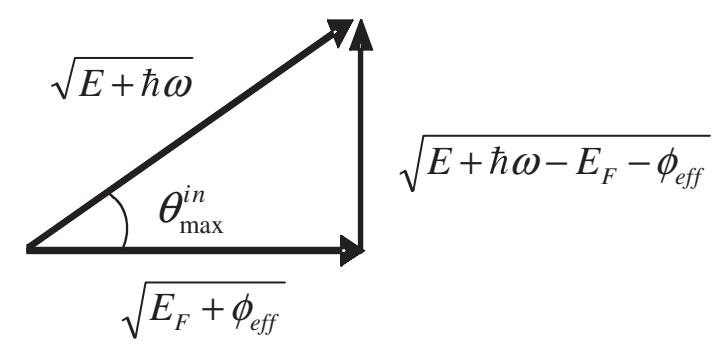

FIG. 4. (Color) Graphical representation of the electron momentum inside the cathode and the maximum angle of escape.

$$
\sin \theta_{\max }^{\text {out }}=\sin \theta_{\max }^{\text {in }} \sqrt{\frac{E+\hbar \omega}{E+\hbar \omega-E_{F}-\phi_{\text {eff }}}} \Rightarrow \theta_{\max }^{\text {out }}=\frac{\pi}{2} .
$$

Electrons which are incident at the maximum escape angle or critical angle, escape the cathode with an external angle of $\pi / 2$. Electrons incident at angles larger than the maximum escape angle are reflected back into the cathode. In the rest of this paper we define $\theta_{\max } \equiv \theta_{\max }^{\text {in }}$ since the maximum angle in the vacuum is always $\pi / 2$.

Reflecting on these relations, it is reasonable to associate these square-root quantities with refractive indices the electron experiences at the cathode-vacuum interface. Therefore we define the following electron indices of refraction:

$$
n_{\text {in }}=\sqrt{E+\hbar \omega}
$$

and

$$
n_{\text {out }}=\sqrt{E+\hbar \omega-E_{F}-\phi_{\text {eff }}}
$$

Introducing the concept of refraction allows us to apply the well-known theory of optical refraction at boundaries to describe emission from angled or curved cathode surfaces. The idea that the emitted electrons can be focused or collimated by the surface is intriguing [12] and its impact on beam dynamics will be investigated in our future studies.

\section{B. Derivation of the photoelectric emittance for metals}

The rms emittance in terms of the moments of the electron distribution is defined as [13]

$$
\varepsilon_{x} \equiv \sqrt{\left\langle x^{2}\right\rangle\left\langle x^{\prime 2}\right\rangle-\left\langle x x^{\prime}\right\rangle^{2}}
$$

with the slope of the electron trajectory given by

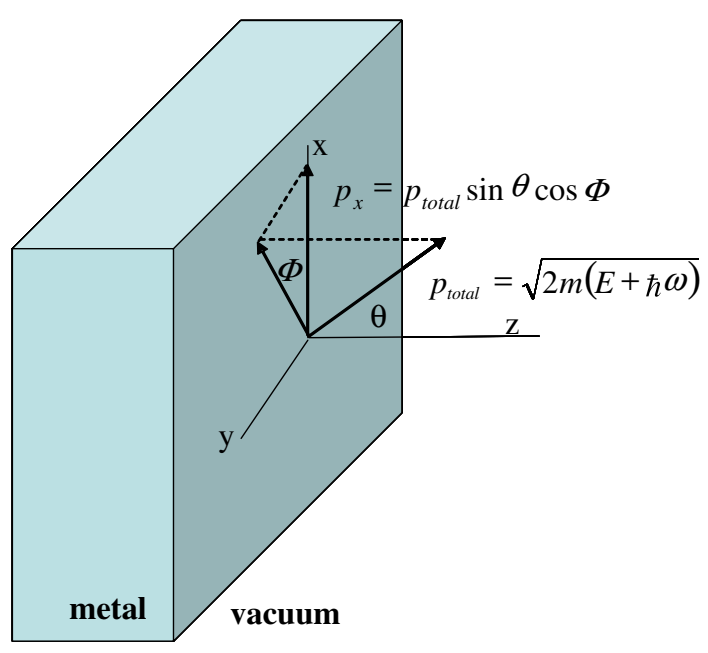

FIG. 5. (Color) Definition of the coordinate system and components of the momentum just inside the cathode surface used to derive the photoelectric emittance. 


$$
x^{\prime}=\frac{d x}{d z}=\frac{d \dot{x}}{d \dot{z}}=\frac{p_{x}}{p_{z}} .
$$

It is generally assumed that since the electrons are rapidly accelerated from rest, their relative velocities are small and the average longitudinal momentum of the ensemble, which we express as $\beta \gamma m c$, can be used in place of $p_{z}$. This assumption is fundamental to the paraxial approximation. Therefore in terms of $x$ and $p_{x}$, the emittance can be written as [14]

$$
\varepsilon_{x}=\frac{1}{\beta \gamma m c} \sqrt{\left\langle x^{2}\right\rangle\left\langle p_{x}^{2}\right\rangle-\left\langle x p_{x}\right\rangle^{2}} .
$$

If we further assume that there is no correlation between the location of emission and the transverse momentum, then the cross term is zero and one obtains the following for the rms normalized emittance:

$$
\varepsilon_{n} \equiv \beta \gamma \varepsilon_{x}=\sigma_{x} \frac{\sqrt{\left\langle p_{x}^{2}\right\rangle}}{m c}
$$

where $\sigma_{x} \equiv \sqrt{\left\langle x^{2}\right\rangle}$. If we define the dimensionless rms transverse momentum, $\sigma_{p_{x}}$, as

$$
\sigma_{p_{x}} \equiv \frac{\sqrt{\left\langle p_{x}^{2}\right\rangle}}{m c}
$$

then the rms normalized emittance becomes simply

$$
\varepsilon_{n}=\sigma_{x} \sigma_{p_{x}}
$$

The momentum components of the excited electron while still inside the metal are given in terms of a coordinate system aligned to the cathode surface as shown in Fig. 5. The variance of the transverse momentum can be computed from the three-step model of photoemission in a manner similar to the quantum efficiency as given above,

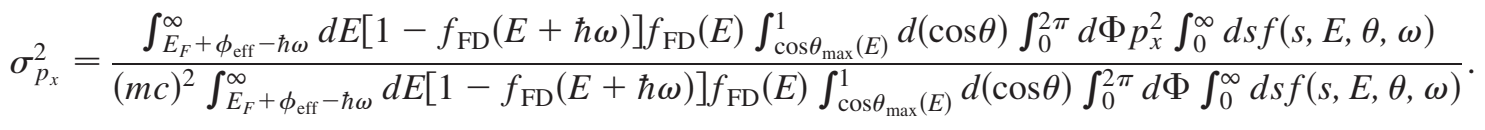

Here again, the Fermi-Dirac function, $f_{\mathrm{FD}}$, gives the density of initial states and $\left[1-f_{\mathrm{FD}}(E+\hbar \omega)\right]$ gives the density of excited states. It is interesting to note that in this case, the electron-electron-scattering function, $f(s, E, \theta, \omega)$, appears in both the numerator and denominator. Applying the same conditions as in the QE derivation, we assume the electron-electron scattering probability only depends upon the photon wavelength and the $F_{e-e}(\omega)$ functions cancel. When the full angular and energy dependence of the electron-scattering function are included the scattering probability does not cancel. However, the effect on the transverse momentum is small and numerical integration results including these effects are described below. We also assume the temperature is $0 \mathrm{~K}$ to simplify the integration and produce an analytic result. The effect of nonzero temperatures is also described below. Replacing the Fermi-Dirac distributions with Heavisidestep functions results in the following expression for the variance of the transverse momentum:

$$
\sigma_{p_{x}}^{2}=\frac{\int_{E_{F}+\phi_{\mathrm{eff}}-\hbar \omega}^{E_{F}} d E \int_{\cos \theta_{\max }}^{1} d(\cos \theta) \int_{0}^{2 \pi} d \Phi p_{x}^{2}}{(m c)^{2} \int_{E_{F}+\phi_{\mathrm{eff}}-\hbar \omega}^{E_{F}} d E \int_{\cos \theta_{\max }}^{1} d(\cos \theta) \int_{0}^{2 \pi} d \Phi}
$$

As described earlier the maximum angle of escape inside the metal, $\theta_{\max }$, is determined by the requirement that the energy perpendicular to the surface exceeds the vacuum state energy,

$$
\frac{p_{z}^{2}}{2 m} \geq E_{F}+\phi_{\text {eff }}
$$

As given by the vector relation in Fig. 4, the maximum cosine angle of emission is

$$
\cos \theta_{\max }=\frac{p_{z}}{p_{\text {total }}}=\sqrt{\frac{E_{F}+\phi_{\text {eff }}}{E+\hbar \omega}}
$$

and the $x$ component of the momentum is

$$
p_{x}=\sqrt{2 m(E+\hbar \omega)} \sin \theta \cos \Phi .
$$

The expression for the variance of the transverse momentum then becomes

$$
\sigma_{p_{x}}^{2}=\frac{2}{m c^{2}} \frac{\int_{E_{F}+\phi-\hbar \omega}^{E_{F}} d E \int_{\cos \theta_{\max }}^{1} d(\cos \theta) \int_{0}^{2 \pi} d \Phi(E+\hbar \omega) \sin ^{2} \theta \cos ^{2} \Phi}{\int_{E_{F}+\phi-\hbar \omega}^{E_{F}} d E \int_{\cos \theta_{\max }}^{1} d(\cos \theta) \int_{0}^{2 \pi} d \Phi} .
$$

Or rewriting with the three integrations explicitly separated and using the expression for the maximum cosine angle 
derived above, we obtain

$$
\sigma_{p_{x}}^{2}=\frac{2}{m c^{2}} \frac{\int_{E_{F}+\phi_{\mathrm{eff}}-\hbar \omega}^{E_{F}}(E+\hbar \omega) d E}{\int_{E_{F}}^{E_{F}}+\phi_{\mathrm{eff}}-\hbar \omega} \frac{\int_{\sqrt{\left(E_{F}+\phi_{\mathrm{eff}}\right) /(E+\hbar \omega)}}^{1} \sin ^{2} \theta d(\cos \theta)}{\int_{\sqrt{\left(E_{F}+\phi_{\mathrm{eff}}\right) /(E+\hbar \omega)}}^{1} d(\cos \theta)} \frac{\int_{0}^{2 \pi} \cos ^{2} \Phi d \Phi}{\int_{0}^{2 \pi} d \Phi} .
$$

The angular integrations can be done separately and are given by

$$
\frac{\int_{0}^{2 \pi} \cos ^{2} \Phi d \Phi}{\int_{0}^{2 \pi} d \Phi}=\frac{1}{2} ; \quad \int \sin ^{2} \theta d(\cos \theta)=\cos \theta-\frac{1}{3} \cos ^{3} \theta
$$

Substituting these integrals into Eq. (34) results in the following expression for the variance of the transverse momentum:

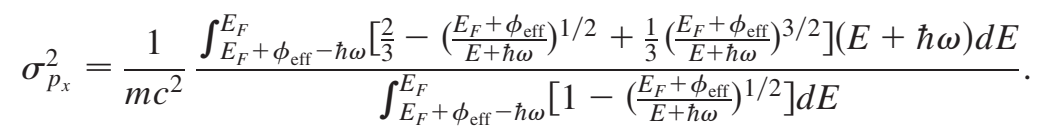

This equation can be analytically integrated and the rms of the dimensionless transverse momentum for photoemission is found to be

$$
\sigma_{p_{x}}=\sqrt{\frac{\hbar \omega-\phi_{\mathrm{eff}}}{3 m c^{2}}}
$$

and therefore the normalized emittance is

$$
\varepsilon_{n}=\sigma_{x} \sigma_{p_{x}}=\sigma_{x} \sqrt{\frac{\hbar \omega-\phi_{\mathrm{eff}}}{3 m c^{2}}}
$$

Figure 6 plots the photoelectric emittance for copper as a function of photon energy for applied electric fields of 0 , 50 , and $100 \mathrm{MV} / \mathrm{m}$ using the parameters given in Table I. The emittance approaches 0 as the photon energy approaches the effective work function and the reduced beam brightness, which we define as the $\mathrm{QE} / \sigma_{p_{x}}^{2}$, also

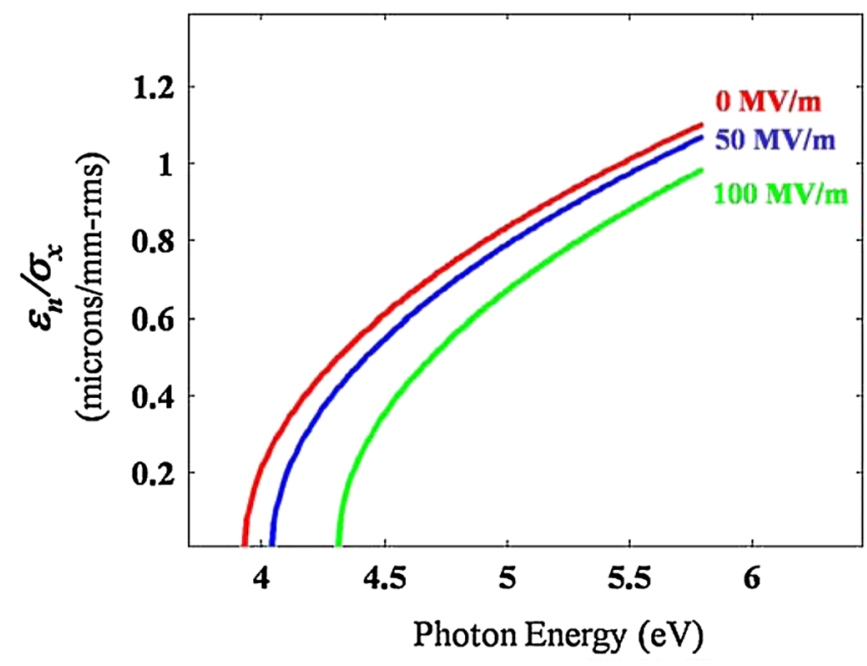

FIG. 6. (Color) The normalized emittance per rms beam size for $\mathrm{Cu}$ as a function of photon energy for the typical operating electric fields of metal cathodes in rf guns due to the Schottky reduction of the barrier as described in the text. The work function is assumed to be $4.31 \mathrm{eV}$. monotonically approaches 0 as the photon energy approaches the effective work function since the QE varies faster than the square of the transverse momentum spread.

The simplifying assumptions used to arrive at this analytic result have a small effect on the transverse momentum spread. We have numerically integrated the energy variable in Eq. (28) using the parameters in Table I to determine the effect of temperature, angular dependence of the electronscattering function, and the energy dependence of the mean-free path on the transverse momentum. Including the $E^{-3 / 2}$ energy dependence of the mean-free path results in a $0.8 \%$ decrease in the transverse momentum spread and including the scattering function angular dependence decreases the rms transverse momentum another $0.2 \%$. As the difference between the photon energy and effective work function increases the error in the approximation will increase.

The transverse momentum is more sensitive to temperature than the $\mathrm{QE}$ at room temperature. At $300 \mathrm{~K}$ the thermal emittance increases by $0.2 \%$ compared to $0 \mathrm{~K}$. At $2000 \mathrm{~K}$ both the QE and rms transverse momentum are increased by approximately $10 \%$.

\section{THE RELATION BETWEEN EMITTANCE AND QUANTUM EFFICIENCY}

With these equations for the emittance and QE it is interesting to investigate the connection between them. In Fig. 7 the quantum efficiency and normalized emittance per rms beam size are plotted as functions of the effective work function. Both the QE and emittance decrease with increasing effective work function suggesting the disappointing result that to reduce the emittance one has to accept a lower QE. This type of analysis illustrates the value of a unified theory of photoemission where consistency demands that the same effective work function be used to compute both the $\mathrm{QE}$ and thermal emittance. For example, a QE of $5 \times 10^{-5}$ has an effective work function of $4.56 \mathrm{eV}$ which gives a theoretical cathode emittance with a slope of 0.46 microns $/ \mathrm{mm}$ (rms). However, recent 


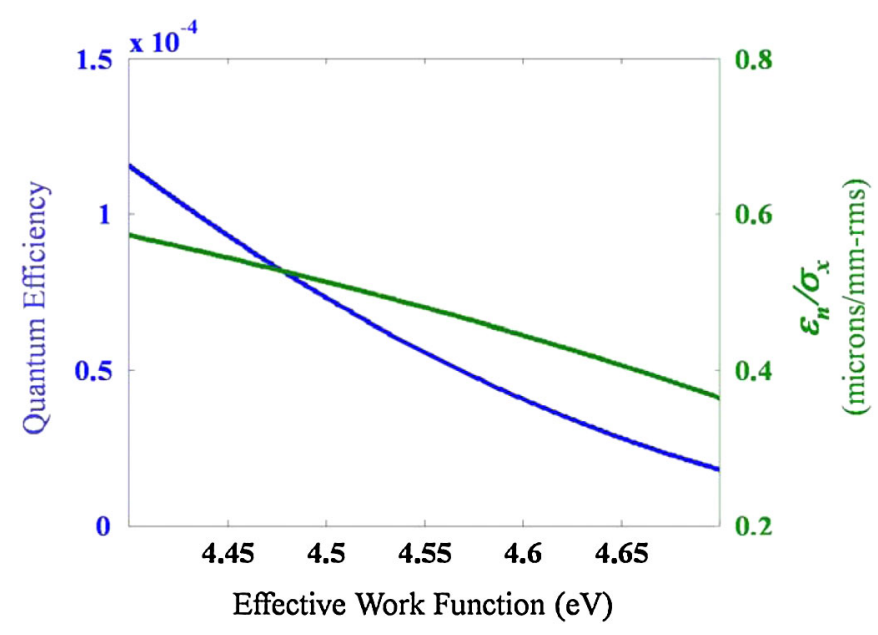

FIG. 7. (Color) The quantum efficiency and the normalized emittance per rms beam size plotted as functions of the effective work function, $\phi_{\text {eff }}$, for a photon energy of $4.90 \mathrm{eV}\left(\lambda_{\text {laser }}=\right.$ $253 \mathrm{~nm})$.

Linac Coherent Light Source (LCLS) measurements on a copper cathode with this QE give an emittance slope approximately twice this theoretical value with similar experimental results previously reported by Brookhaven National Laboratory (BNL) [15], Sumitomo [16], and Stanford Linear Accelerator Center (SLAC) [4,17]. In general, the experimental thermal emittance as measured in an rf photocathode gun is a factor of 2 larger than the theoretical thermal emittance.

The quantum efficiency can be thought of as the percentage of electrons inside the metal with sufficient longitudinal momentum to overcome the effective work function. It is interesting to note that any changes to the transverse momentum of the electrons inside the metal would have no effect on the QE. Thus, the QE is a measure of the longitudinal momentum distribution.

The thermal emittance is by definition a measure of the transverse momentum of the electrons that are emitted. At first glance it may not appear that the $\mathrm{QE}$ and thermal emittance are linked since they depend on orthogonal components of momentum. Although the longitudinal momentum does not have a direct influence on the thermal emittance, it does determine which electrons are emitted and thus which electrons' transverse momentum contribute to the emittance. One of the reasons metals have a relatively low thermal emittance is that the electrons with significant transverse momentum are not emitted. Because of the work function, only electrons moving nearly normal to the surface are emitted. This is why the width of the transverse momentum distribution decreases as the effective work function increases. Thus, electrons with low transverse momentum are selectively emitted.

It is clearly desirable to maximize the $\mathrm{QE}$ and minimize the thermal emittance but due to their interdependence this is difficult. However, if one could shift electrons from the
Fermi energy towards the minimum possible emission energy (see Fig. 2), the thermal emittance would be reduced since the emitted electrons have less available transverse momentum. In this work, we have assumed a constant density of states near the Fermi energy. Distributions with densities that increase as the energy decreases below the Fermi energy can increase the QE and simultaneously decrease the thermal emittance. It is interesting to note that increasing the cathode temperature shifts electrons from just below the Fermi energy to just above the Fermi energy resulting in higher thermal emittance.

The only way to alter the QE with no effect on the thermal emittance is via variation of the reflectivity, optical absorption depth, or the electron mean-free path since to first order the scattering probability cancels in the expression for the transverse momentum. Thus, in principle, one could select a cathode material with the optimal complex optical index of refraction to minimize both the reflectivity and the optical absorption depth. However, the maximum increase in the $\mathrm{QE}$ for $\mathrm{Cu}$ with this method is limited to approximately a factor of 5 since the reflectivity is approximately $30 \%$ and the reduction due to scattering is roughly a factor of 3 . One must be careful not to choose an optical wavelength to achieve the desirable optical parameters which cause a larger decrease in QE or an unacceptable increase in the thermal emittance due to the change in photon energy or mean-free path.

\section{COMPARISON WITH OTHER EMITTANCE CALCULATIONS}

As mentioned earlier, the calculation of the thermal emittance for $\mathrm{Cs}_{2} \mathrm{Te}$ cathodes was performed by Floettmann in which the three-step model was introduced with an average for the electron energy distribution. Later calculations, including the one presented here, all follow the same basic assumption of the three-step model with a Fermi-Dirac function at zero temperature with various refinements, e.g., the Schottky potential has been included. Here we review the published expressions for the photoemission emittance, and describe their differences and similarities with each other and the results of this paper.

The thermal emittance derivation by Floettmann for $\mathrm{Cs}_{2} \mathrm{Te}$ semiconductor cathodes is given as $[11,18]$

$$
\varepsilon_{\mathrm{th}}=\sigma_{x} \sqrt{\frac{2 E_{K}}{3 m c^{2}}} .
$$

Floettmann has assumed a delta function energy distribution, which is valid for CsTe, to derive this expression. Defining $E_{K}$ as the average energy for emission from metals gives

$$
E_{K}=\frac{\hbar \omega-\phi_{\mathrm{eff}}}{2}
$$


which makes our relation for the photoelectric emittance identical to that of Floettmann's.

Clendenin and Mullhollan [19] followed a similar approach but included the Schottky potential to the material work function which is significant for metals. Their model has two important differences with our model. They used a delta function energy distribution at the Fermi energy and they assumed the Fermi energy was at $0 \mathrm{eV}$. The delta function energy distribution makes the emittance increase and the Fermi energy at zero causes the emittance to decrease. The net result is the two assumptions nearly cancel so the numerical result they obtain for $\mathrm{Cu}$ is very similar to our result although the equation is significantly different.

While mathematically generalized to cover thermionic, field emission, and photoemission, the same basic derivation is given in a recent book by Jensen [20]. In it he uses quantum mechanical distribution function approach but applies essentially the same physical approximations as described above. However, he obtains a different result, namely,

$$
\varepsilon_{n, \mathrm{rms}}=\sigma_{x} \sqrt{\frac{E_{F}\left(\hbar \omega-\phi_{\mathrm{eff}}\right)}{3 m c^{2}\left(\hbar \omega+E_{F}\right)}}
$$

With parameters from Table I, this expression gives approximately $25 \%$ lower emittance than Eq. (38). Careful reading of his analysis shows the discrepancy originates with his using the initial electron energy before absorbing the photon to compute the transverse momentum. Or specifically, in the numerator of Eq. (36), the factor $E d E$ is used instead of $(E+\hbar \omega) d E$. This statement is clarified by comparing our expression for the transverse momentum variance, Eq. (36), with Jensen's written in terms of this paper's variables,

$$
\sigma_{p_{x}}^{K J^{2}}=\frac{1}{m c^{2}} \frac{\int_{E_{F}+\phi_{\mathrm{eff}}-\hbar \omega}^{E_{F}}\left[\frac{2}{3}-\left(\frac{E_{F}+\phi_{\text {eff }}}{E+\hbar \omega}\right)^{1 / 2}+\frac{1}{3}\left(\frac{E_{F}+\phi_{\text {eff }}}{E+\hbar \omega}\right)^{3 / 2}\right] E d E}{\int_{E_{F}+\phi_{\text {eff }}-\hbar \omega}^{E_{F}}\left[1-\left(\frac{E_{F}+\phi_{\text {eff }}}{E+\hbar \omega}\right)^{1 / 2}\right] d E} .
$$

This expression and Eq. (36) use the same maximum angle of escape, $\cos ^{-1}\left(\sqrt{\frac{E_{F}+\phi_{\text {eff }}}{E+\hbar \omega}}\right)$, confirming that $E$ indeed represents the electron energy before photon absorption in both cases. Thus Eq. (42) displays an inconsistency between the energy $E$ used for the maximum escape angle and that used to compute the momentum.

\section{DISCUSSION AND SUMMARY}

This paper derives expressions for the quantum efficiency and thermal emittance of photoemission from metal cathodes. This was done assuming the electron energy distribution is given by the Fermi-Dirac function at zero temperature. The emission process follows the three-step model which was used consistently to obtain the QE and emittance expressions. The second step of drifting to the surface includes electron-electron scattering which is numerically estimated to be less than a percent effect on the emittance.

There are many phenomena which were not discussed in this paper. For example, the model used here assumes emission from the volume of the cathode and ignores any polarization effects [21] except those contained in the Fresnel relations for the reflectivity and optical attenuation length. In addition the effects of surface roughness are ignored. The phenomenon related to emission from a tilted surface and the associated transverse energy gain from the transverse component of the applied field is not included. Nor was field enhancement due to isolated surface protrusions considered. These and other effects will increase the thermal emittance. Useful work has been published on these topics of surface roughness [22,23]; however, at this time it is not known whether one or some combination of these effects is important. However, given that the observed emittance is generally larger than that given by these formulas necessitates the application of some or all of the effects just described and will be addressed in a future publication.

In conclusion, this paper presents expressions for the quantum efficiency and the thermal emittance for metal cathodes using a consistent theory of photoemission. The assumptions and approximations were described with some detail and the magnitude of these approximations numerically quantified. Finally we stress the connection between quantum efficiency and the photoelectric emittance and the need to study them with a consistent analysis.

\section{ACKNOWLEDGMENTS}

This paper has benefited from stimulating discussions with Klaus Floettmann (DESY), Sven Lederer (DESY), Kevin Jensen (NRL), John Smedley (BNL), Axel Brachmann (SLAC), and Dao Xiang (SLAC). This work was supported by the U.S. Department of Energy under Contract No. DE-AC02-76SF00515.

[1] H. Puff, Phys. Status Solidi 4, 125 (1964).

[2] C. N. Berglund and W.E. Spicer, Phys. Rev. 136, A1030 (1964).

[3] A.H. Sommer, Photoemissive Materials: Preparation, Properties and Uses (John Wiley \& Sons, Inc., New York, 1968), pp. 5, 21-26. 
[4] J.F. Schmerge et al., in Proceedings of the FEL2004 Conference, http://accelconf.web.cern.ch/AccelConf/ index.html, pp. 205-208; SLAC Report No. SLAC-PUB10763.

[5] D. H. Dowell et al., Phys. Rev. ST Accel. Beams 9, 063502 (2006); SLAC Report No. SLAC-PUB-11788.

[6] K. L. Jensen et al., Appl. Phys. Lett. 89, 224103 (2006).

[7] W. E. Spicer, Phys. Rev. Lett. 11, 243 (1963).

[8] W. E. Spicer, Phys. Rev. 154, 385 (1967).

[9] L. H. Hall et al., Phys. Rev. 95, 559 (1954).

[10] R. H. Fowler, Phys. Rev. 38, 45 (1931).

[11] K. Floettmann, TESLA FEL, Reports 1997-01, 1997, http://flash.desy.de/sites/site_vuvfel/content/e403/e1642/ e839/e829/infoboxContent830/tesla-fel1997-01.pdf.

[12] D. Dowell, S. Lidia, and J. Schmerge, Lecture 2: Electron Emission and Cathode Emittance, USPAS Course on High Brightness Electron Injectors for 4th Generation Light Sources, UC Santa Cruz, 2008 Santa Rosa, CA, http:// uspas.fnal.gov/materials/High_Brightness_Electron_ Injectors_for_Light_Sources.html.

[13] M. Reiser, Theory and Design of Charged Particle Beams (John Wiley \& Sons, Inc., New York, 1994) pp. 57, 358360 , and references therein.

[14] F. J. Sacherer, IEEE Report No. IEEE NS-18, 1971, pp. 1105-1107.
[15] W. Graves et al., in Proceedings of the 19th Particle Accelerator Conference, Chicago, Illinois, 2001 (IEEE, Piscataway, NJ, 2001), pp. 2227-2229.

[16] J. Yang et al., in Proceedings of the 8th European Particle Accelerator Conference, Paris, 2002 (EPS-IGA and CERN, Geneva, 2002), pp. 1828-1830.

[17] D. H. Dowell et al., Nucl. Instrum. Methods Phys. Res., Sect. A 507, 327 (2003); SLAC Report No. SLAC-PUB9540.

[18] V. Miltchev et al., in Proceedings of the FEL2005 Conference, http://accelconf.web.cern.ch/AccelConf/ index.html, pp. 560-563.

[19] J. Clendenin and G. Mullhollan, SLAC Report No. SLACPUB-7760; LCLS Report No. LCLS-TN-98-1, 1998.

[20] K. L. Jensen, Advances in Imaging and Electron Physics, Electron Emission Physics, Volume 149 (Academic Press, New York, 2007), pp. 274-276.

[21] D. Xiang et al., Nucl. Instrum. Methods Phys. Res., Sect. A 562, 48 (2006).

[22] M. Krasilnikov, in Proceedings of the FEL2006 Conference, http://accelconf.web.cern.ch/AccelConf/ index.html, pp. 583-586.

[23] D. Xiang et al., in Proceedings of the 2007 Particle Accelerator Conference, Albuquerque, New Mexico, 2007 (IEEE, Albuquerque, New Mexico, 2007), pp. 1049-1051. 